\title{
Utilizing Wiltse Approach for Minimal Access Posterolateral Lumbar Stabilization
}

\author{
Hamdy Mohammed Behairy \\ Department of Neurosurgery, Al-Azhar University, Cairo, Egypt \\ Email: dr.hamdybehairy@hotmail.com
}

How to cite this paper: Behairy, H.M (2018) Utilizing Wiltse Approach for Minimal Access Posterolateral Lumbar Stabilization. Open Journal of Modern Neurosurgery, 8, 101-108.

https://doi.org/10.4236/ojmn.2018.81008

Received: December 16, 2017

Accepted: January 21, 2018

Published: January 24, 2018

Copyright $\odot 2018$ by author and Scientific Research Publishing Inc. This work is licensed under the Creative Commons Attribution International License (CC BY 4.0).

http://creativecommons.org/licenses/by/4.0/

(c) (i) Open Access

\begin{abstract}
An aggressive separation and prolonged overstretching of the paraspinal muscles in the posterior midline approach during lumbar non fusion dynamic stabilization in cases of spondylolisthesis resulting from pars interarticularis fracture may result in postoperative flat back deformity and intractable chronic pain. It is hypothesized that utilizing Wiltse paraspinal inter-muscular approach for this purpose may result in reduction of operative time, protection of integrity, vascularity, nerve supply and strength of paraspinal muscles, shortening of hospitalization and minimizing development of chronic postoperative back pain. So, I have performed this prospective descriptive study that involved 24 patients having single level lumbar instability at L4-5 or L5-S1 levels. All patients were operated upon using Wiltse minimal access posterolateral surgical technique for non-fusion dynamic stabilization. The final results revealed that males were $66 \%$, females were $33 \%$ and ages were 42 \pm 6 years. Trauma was reported in $12 \%$. Low back pain and tenderness were reported in $100 \%$ and root affection reported in $25 \%$. Plain X-ray and MRI were done in $100 \%$ and C.T. was done in $8 \%$ of cases. Level L4-5 was affected in $17 \%$ while level L5-S1 was affected in $83 \%$. Anterolisthesis "grades 0 " was found in $8 \%$, grades 1 in $88 \%$ and retrolisthesis in $4 \%$ of cases. Operative time was 1 hour $\pm 10 \mathrm{~min}$., blood loss was $60 \pm 20 \mathrm{ml}$., patients ambulation was after 6 - 8 hours, hospital stay was 12 - 24 hours. None of cases were complicated with infection, screw loosening, or fixation system break. Back pain VAS diminished from 7 preoperative to 5 in $2^{\text {nd }}$ day, then became 4 by 1 week, 3 by 1 month and 0 by 6 months. It was concluded that utilizing Wiltse approach for posterolateral lumbar stabilization minimizes tissue damage and improves the speed of recovery and outcome.
\end{abstract}

\section{Keywords}

Lumbar Spine Instability, Posterolateral Wiltse Approach, Minimal Access Lumbar Stabilization 


\section{Introduction}

The traditional posterior midline approach to the lumbar spine requires complete separation of the paraspinal muscles from their attachment points on spinous processes and lamina. This extensive muscle stripping could easily damage the posteromedial branches of the spinal nerves and the descending posterior lumbar artery branches. Also, the prolonged overstretching of the paraspinal muscles by the self-retaining retractors to expose the location of entry points for insertion of pedicle screws could result in atrophy and ischemic necrosis of these muscles. Indeed, such damage when accompanied with postoperative scar tissue formation may result in postoperative flat back deformity and intractable chronic pain [1]. For avoiding such complications, Wiltse approach was developed. In 1968, Wiltse proposed postero-lateral paraspinal intramuscular approach to the lumbar spine to perform discectomy for far lateral disc herniation without interruption of healthy intact midline structures. In his original report on his approach, Wiltse described splitting sacrospinalis about two-finger breadths lateral to the midline. He noted that the muscle fibers do not split cleanly since at this level they run in various directions [2]. Later on, he modified his approach to sacrospinalis-splitting using blunt dissection through cleavage plane in the natural gap between multifidus and longissimus paraspinal muscles (Figure 1) which is about $4 \mathrm{~cm}$ lateral to the midline at the avascular interface to markedly reduce muscle tissue damage [4] [5].

The advantages of this approach are; minimal tissue trauma [6], significant reduction of intraoperative muscle retraction [7], marked reduction of operative time, protection of integrity, vascularity, nerve supply and strength of paraspinal muscles, shortening of hospitalization and minimizing development of chronic postoperative back pain [8]. There are two disadvantages of this approach. First is creation of small operating space which could be compensated by magnifica-

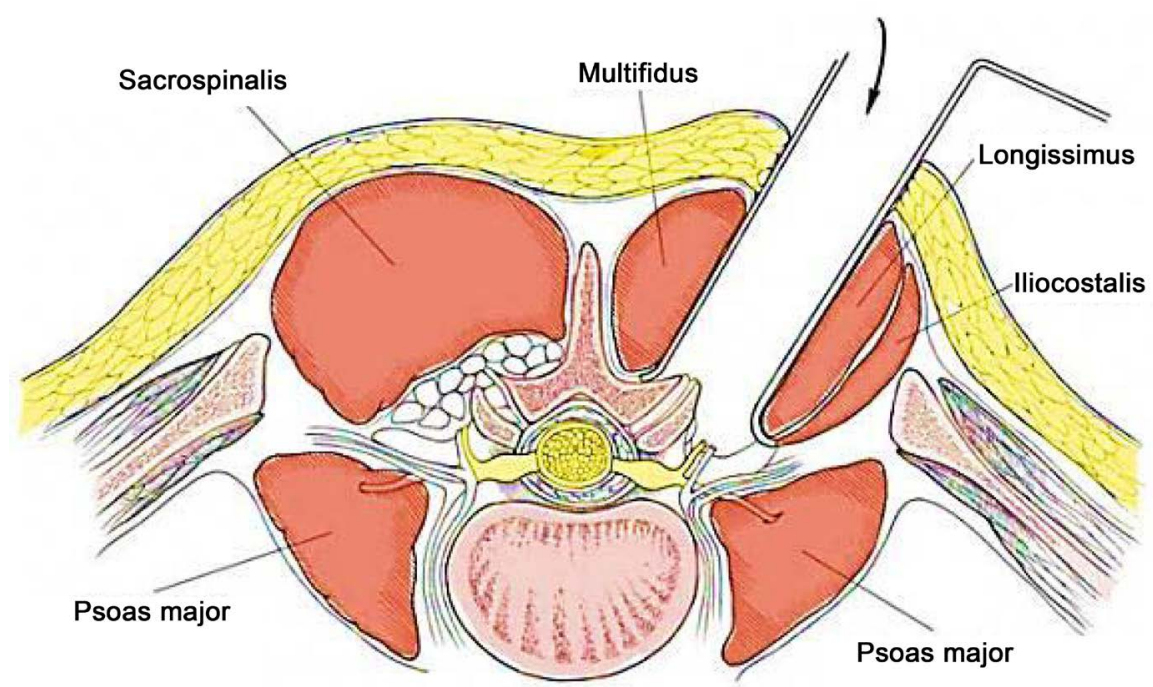

Figure 1. An illustration showing the modified Wiltse muscle-sparing approach through the plane between the multifidus and longissimus muscles [3]. 
tion through wearing loop or better by using the operative microscope. The second is that the surrounding anatomic landmarks are less obvious than those in the traditional approach. So, the transverse process could be mistaken for the facet process, thus damaging smaller blood vessels and increasing bleeding. Prevention of that depends on clear intraoperative imaging and the experience of the surgeon.

In recent years, percutaneous non fusion dynamic fixation technique for management of spondylolisthesis resulting from pars interarticularis fracture was known to have better effects in preserving paraspinal muscles than traditional midline approach with aggressive muscle separation. However, compared to Wiltse approach, percutaneous techniques are associated with higher doses of radiation exposure without better therapeutic outcomes [8].

In comparison with fusion surgery, nonfusion dynamic stabilization is more advantageous; being simpler, safe procedure "especially in elderly patients", reduces or prevents facet joint degeneration and improves clinical outcome [9].

Based on the fore mentioned literature, I performed this study to evaluate the possible replacement of the mostly used muscle destructive midline approach for lumbar stabilization by the less invasive muscle preserving Wiltse approach that was primarily designed for far lateral discectomy aiming to provide an effective management beyond the conservative treatment that often fail in such almost progressive lumbar instability.

\section{Patients and Methods}

This is a prospective descriptive study, performed over 2 years "between September 2015 and September 2017" on 24 patients admitted and managed in Al-Azhar university hospitals, Cairo, Egypt. Patients included in the study are those with single level L4-5 or L5-S1 instability "spondylolysis, spondylolisthesis or retrolisthesis" due to bilateral fractured bars inter-articularis (grades 0,1 or 2) of any age and sex with or without history of trauma were provided no true big disc prolapse or primary lumbar canal stenosis. Patients excluded from the study were those with multiple levels or single level $3^{\text {rd }}$ degree spondylolisthesis and those with true big disc prolapse indicated for discectomy or primary lumbar canal stenosis indicated for decompression. All patients had symptoms of lumbar instability in the form of low back pain increases on sitting and standing and radiates to one or both lower limbs with neurogenic claudication pain in some of them resulting from lumbar canal stenosis secondary to instability. Plain x-ray was done for all patients and revealed bars inter-articularis fracture (spondylolysis) with or without upper vertebral body displacement according to degree of listhesis. MRI T1 and T2, sagittal and axial views revealed the detailed anatomy and pathology of the disc and spinal canal. An informed consent was signed by all participant patients. Wiltse posterolateral surgical technique was used for all patients to perform minimal access non fusion dynamic fixation using transpedicular screws and rods without muscle or muscular vascularity destruction with 
preservation of facet joint and capsule and without opening the spinal canal.

Surgical steps (Table 1); General anesthesia, prone position, radiographic marking of the pedicles, two longitudinal short skin incisions, one on each side 3 $\mathrm{cms}$ long and 3 - $4 \mathrm{cms}$ away from the midline. The lumbar fascia and muscle sheath were opened along the skin incision. The fascia forming the septum between longissimus and multifidus was searched for where the gap between the multifidus and longissimus muscles was isolated then finger was used for gentle blunt separation medial to the fascia. According to [10], the cleavage plane follows some regular pattern in different segments with maximum curvature at L3 segment and minimum curvature and more linear direction at L5 and S1, So, MRI revision intraoperative helps in avoiding inaccuracies. Tubular [11] or narrow blade self-retaining retractor (as Claward retractor used in anterior cervical micro-discectomy) was used to maintain the inter-muscular plane with shorter blade medially over lateral edge of the lamina and longer blade laterally over the transverse process. The facet joints and transverse processes were well identified. Antro-posterior fluoroscopy helps in defining lateral edge of the target pedicle. Lateral fluoroscopy with clamp placed on the transverse process confirms its level. The facet joint capsules and muscle attachments were carefully preserved. Exact puncture point was identified then cortical bone puncture was made by owl perforator then pedicle finder was inserted under imaging guidance. The oblique angle of the inter-muscular gab was used as a direction when inserting the pedicle screw. At L5-S1 level, to avoid iliac crest, the retractor was positioned little more medially than usual and the lateral aspect of the facet joint is drilled and removed. Four mono-axial pedicle screws 35,40 and $45 \mathrm{~mm}$ in length and 6.5 and $7.5 \mathrm{~mm}$ in diameter were used. Screws were driven into the upper and

Table 1. Showing radiological images and operative steps.

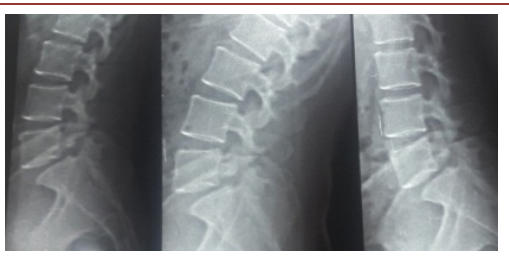

1) Preoperative dynamic plain X-ray showing instability and bilateral pars fracture.



4) Intraoperative photo showing screws and rod fixation.

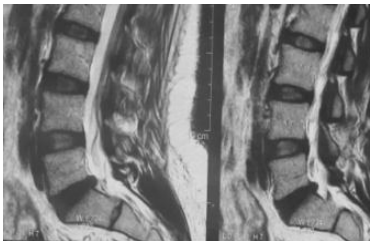

2) Preoperative sagittal T2 MRI showing small L5-S1 disc prolapsed.

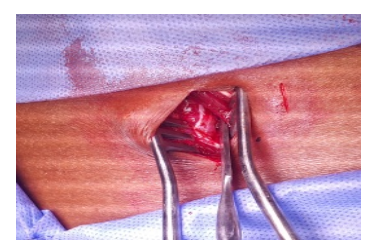

3) Intraoperative photo showing the plain between multifidus and longissimus muscles.

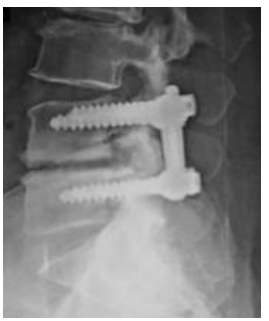

7) Follow up lateral plain $\mathrm{x}$-ray for a case with L4-5
5) Intraoperative fluoroscopy at the end of the procedure. stabilization.
6) Bilateral paramedian closed wounds.

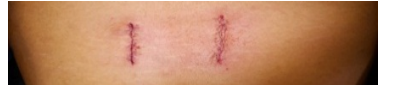


lower vertebrae then the pre-bent connecting rod was implanted and the pedicle screws were tightened after performing suitable distraction. It was important to note that screw placement without disturbing the facet capsule and joint is very critical. After final imaging, the wound was closed in layers without need for drains. Skin was closed by subcuticular stitches and covered with sterile dressing for 5 days. The operative time, intraoperative blood loss and postoperative ambulation time were calculated. Wounds were followed up till complete healing. Wound complications and their management were recorded. Plain X-ray and/or CT scan was considered if any instance in which the status of a screws was questionable. The visual analogue scale (VAS) was used to evaluate back pain improvement. VAS scores were ranged from 0 to 10 points with 0 point indicates complete lack of pain and 10 points indicate unbearable pain. VAS was recorded preoperative and postoperative after 1 day, 1 week, 1 month, 3 months and 6 months. Fixation system integrity was followed up for all through the follow up periods that were from 6 months to 2 years. All patients were operated upon during the first one and half years of the study while the last 6 months period was for follow up of the last case.

\section{Results}

This is a prospective descriptive study performed over 2 years, between September 2015 and September 2017 and involved 24 patients admitted and operated upon at Al-Azhar university hospitals, Cairo, Egypt. There were 16 males (66 \%) and 8 females $(33 \%)$. The ages were $42 \pm 6$ years. History of trauma was found in 3 patients (12\%) while in 21 patients $(88 \%)$ no definite trauma was mentioned. The preoperative symptoms included low back pain in all cases (100\%) that was exaggerated on sitting, standing and with movement. Much exaggeration was found on bending forward or after carrying heavy objects. There was intermittent radicular pain along sciatic nerve, unilaterally in 8 cases (33\%) and bilaterally in 2 cases $(8 \%)$ where pain forced the patient to stop from time to time while walking as a neurogenic claudication pain. No other preoperative symptoms. Preoperative signs included low back tenderness in all cases. Signs of root affection in the form of straight leg raising limitation, was positive in 6 cases (25\%). There were no motor, sensory or reflexes abnormalities. Radiological investigations included plain X-ray and MRI lumbosacral spine for all cases $(100 \%)$. C.T. scan lumbosacral spine was done for 2 cases (8\%) of grade 0 spondylolisthesis (spondylolysis) for more declaration of pars interarticularis fracture. Imaging revealed single level L4-5 or L5-S1 bilateral fractured bars interarticularis. Level L4-5 was affected in 4 cases (17\%) while level L5-S1 was affected in 20 cases (83\%). Spondylolysis (grades 0) was found in 2 cases (8\%), spondylolisthesis grades 1 was found in 21 cases $(88 \%)$, no cases of higher degrees of spondylolisthesis and 1 case of retrolisthesis (4\%).

All cases were operated upon via Wiltse minimal access posterolateral surgical technique for non-fusion dynamic stabilization using four mono-axial pedicle 
screws. The operative time was 1 hour $\pm 10 \mathrm{~min}$. Intraoperative blood loss was $60 \pm 20 \mathrm{ml}$. Postoperative patient' ambulation was after 6 - 8 hours. Hospital stay was 12 - 24 hours. Follow up durations was 6 months to 2 years. There were no vascular or neurological complications, wound infection, screw loosening or misplacement, or fixation system break. As the back pain was the main or the only problem of all patients in the study, it was evaluated using the visual analogue scale (VAS) with scores ranged from 0 point (no pain) to 10 points (intolerable pain). VAS was recorded preoperative and postoperative at 1 day, 1 week, 1 month, 3 months and 6 months. It was found that back pain started to diminish in $2^{\text {nd }}$ day, became markedly tolerable by 1 week, became minimal by 1 month and disappeared by 3 - 6 months (Table 2).

As the number of patients in this study is small which is a weak point in this study, it is recommended to perform further studies further experience and multicenter collaboration on bigger numbers of patients with different variables in a comparative form as in cases associated with significant disc prolapse or lumbar canal stenosis, cases with multiple levels affection and for different associating pathologies to elucidate different drawbacks.

\section{Discussion}

Several authors have described paraspinal muscle damage seen after midline muscle-stripping approaches [4]. Since its introduction in 1988, microsurgery via Wiltse paraspinal approach has been regarded as a principal surgical option for management of lumbar foraminal or extraforaminal lesions. Modified Wiltse approach enables working through natural muscle gaps without special technical equipment, preserves integrity of paraspinal muscles and midline structures and significantly reduces surgical tissue-destructive trauma and bleeding [12].

It was agreed (on basis of cadaver studies) that the splitting should be done at the natural cleavage plane between the multifidus and the longissimus parts of the sacrospinalis [5].

Trans-pedicle screw fixation produces good fracture reduction and firm fixation. This approach is an oblique one that leads itself to the correct trajectory for insertion of appropriate screws so avoids nerve injury. It facilitates an earlier ambulation, reduces hospitalization time and postoperative back pain and consequently improves the final outcome.

Table 2. Shows VAS for pre and postoperative back pain evaluation.

\begin{tabular}{cc}
\hline Timing for evaluation of back pain & Range of VAS \\
\hline Before surgery & $4-9$ \\
1 day postoperative & $4-7$ \\
1 week postoperative & $3-5$ \\
1 month postoperative & $0-4$ \\
3 months postoperative & $0-2$ \\
6 months postoperative & 0 \\
\hline
\end{tabular}


In comparison with fusion surgery, nonfusion dynamic stabilization was advantageous as it reduced or prevented facet joint degeneration and improved clinical outcomes [9].

\section{Conclusion}

Utilizing Wiltse approach for posterolateral inter-muscular lumbar non fusion dynamic stabilization in cases of lower lumbar low degree instability is recommended as it was found to minimize tissue damage that often occur in midline approach and improve the speed of recovery and outcome.

\section{Acknowledgements}

I acknowledge all members of my neurosurgery department, Al-Azhar University for support all through this study.

\section{Conflicts of Interest}

There are no conflicts of interest.

\section{References}

[1] Spoor, A. and Oner, F. (2013) Minimally Invasive Spine Surgery in Chronic Low Back Pain Patients. Journal of Neurosurgical Sciences, 57, 203-218.

[2] Wiltse, L.L., Bateman, J.G., Hutchinson, R.H. and Nelson, W.E. (1968) The Paraspinal Sacrospinalis-Splitting Approach to the Lumbar Spine. Journal of Bone and Joint Surgery American, 50, 919-926. https://doi.org/10.2106/00004623-196850050-00004

[3] Anand, N., Baron, E. and Bray, R. (2007) Benefits of the Paraspinal Muscle-Sparing Approach versus the Conventional Midline Approach for Posterior Nonfusion Stabilization: Comparative Analysis of Clinical and Functional Outcomes. SAS Journal, 1, 93-99. https://doi.org/10.1016/S1935-9810(07)70053-1

[4] Kim, C.W. (2010) Scientific Basis of Minimally Invasive Spine Surgery: Prevention of Multifidus Muscle Injury during Posterior Lumbar Surgery. Spine (Phila Pa 1976), 35, S281-286. https://doi.org/10.1097/BRS.0b013e3182022d32

[5] Vialle, R., Wicart, P., Drain, O., Dubousset, J. and Court, C. (2006) The Wiltse Paraspinal Approach to the Lumbar Spine Revisited: An Anatomic Study. Clinical Orthopaedics and Related Research, 445, 175-180.

[6] Li, H., Yang, L., Xie, H., Yum, L., Wei, H. and Cao, X. (2015) Surgical Outcomes of Mini-Open Wiltse Approach and Conventional Open Approach in Patients with Single-Segment Thoracolumbar Fractures without Neurologic Injury. Journal of Biomedical Research, 29, 76-82.

[7] Mahar, A., Kim, C., Wedemeyer, M., Mitsunaga, L., Odell, T., Johnson, B. and Garfin, S. (2007) Short-Segment Fixation of Lumbar Burst Fractures Using Pedicle Fixation at the Level of the Fracture. Spine (Phila Pa 1976), 32, 1503-1507. https://doi.org/10.1097/BRS.0b013e318067dd24

[8] Anekstein, Y., Brosh, T. and Mirosky, Y. (2007) Inter-Mediate Screws in Short Segment Pedicular Fixation for Thoracic and Lumbar Fractures: A Wiltse Approach for Thoracolumbar Fractures. International Journal of Clinical and Experimental Medicine, 9, 13733-13742. https://doi.org/10.1097/01.bsd.0000211240.98963.f6 
[9] Lee, S., Jahng, T. and Kim, H. (2016) Facet Joint Changes after Application of Lumbar Nonfusion Dynamic Stabilization. Neurosurgical Focus, 40, E6. https://doi.org/10.3171/2015.10.FOCUS15456

[10] Palmer, D., Allen, J., Williams, P., Voss, A., Jadhav, V., Wu, D. and Cheng, W. (2011) Multilevel Magnetic Resonance Imaging Analysis of Multifidus-Longissimus Cleavage Planes in the Lumbar Spine and Potential Clinical Applications to Wiltse's Paraspinal Approach. Spine (Phila Pa 1976), 36, 1263-1267.

https://doi.org/10.1097/BRS.0b013e3181f520e8

[11] Lee, Y., Cha, J. and Park, J. (2010) Clinical Outcome of Minimally Invasive Tubular Retractor Assisted Microscopic Discectomy in Far Lateral Lumbar Disc Herniation. Kor J Spine, 7, 155-160. https://doi.org/10.1097/00003086-197303000-00009

[12] Wiltse, L.L. (1973) The Paraspinal Sacrospinalis-Splitting Approach to the Lumbar Spine. Clinical Orthopaedics and Related Research, No. 91, 48-57. 